\title{
Prenatal Anhydramnios in a Girl: An Exceptional Case of Obstruction by Bilateral Ectopic Ureter
}

ISSN: 2576-9200

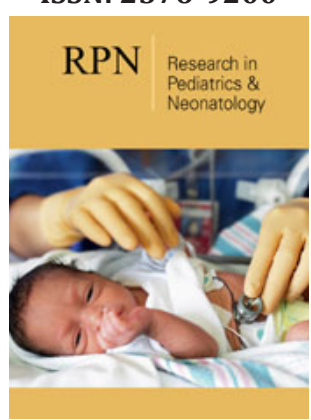

*Corresponding author: Beatriz Fernández-Bautista, Pediatric surgeon, Gregorio Marañon University Hospital, Spain

Submission: 潐 December 08, 2020

Published: 䟧January 28, 2021

Volume 5 - Issue 3

How to cite this article: Beatriz Fernández-Bautista, Jose María Angulo, Laura Burgos, Alberto Parente, Javier Ordóñez, Rubén Ortiz. Prenatal Anhydramnios in a Girl: An Exceptional Case of Obstruction by Bilateral Ectopic Ureter. Research in Pediatrics \& Neonatology. 5(3). RPN. 000611. 2021. DOI: $10.31031 /$ RPN.2021.05.000611

Copyright $(\subset$ Beatriz Fernández-Bautista. This article is distributed under the terms of the Creative Commons Attribution 4.0 International License, which permits unrestricted use and redistribution provided that the original author and source are credited.

\author{
Beatriz Fernández-Bautista ${ }^{1 *}$, Jose María Angulo ${ }^{1}$, Laura Burgos ${ }^{1}$, Alberto \\ Parente $^{1}$, Javier Ordóñez ${ }^{2}$ and Rubén Ortiz ${ }^{1}$ \\ ${ }^{1}$ Pediatric Surgeon, Gregorio Marañon University Hospital, Spain \\ ${ }^{2}$ Resident of Pediatric Surgery, Gregorio Marañón University Hospital, Spain
}

\begin{abstract}
Introduction: The diagnosis of oligoamnios or anhydramnios is usually associated with male fetuses, being the most frequent urological cause the posterior urethral valves and the ureterocele in second place.We present an exceptional case of anhydramnios in a female fetus secondary to obstruction of a bilateral ectopic ureter with severe worsening of renal function.
\end{abstract}

Material and methods: We present the case of a preterm newborn (33+6 weeks) with a prenataldiagnosis in of bilateral ureterohydronephrosis with double left system. Labor was induced at 33 weeks due to anhydramnios.Progressive clinical, analytical and radiological worsening lead to the need of cystoscopy at nine days of life. The cystoscopic study showed a right ectopic ureter that imprinted on the vagina but whose meatus was not found, a ureter of the left upper kidney leading to the urethra, and the imprint of the left lower kidney ureter in the bladder. With electrocoagulation, a bilateral transurethral neo-orifice (TUNO) is performed as a drainage method of both dilated systems.

Results: In the postoperative period diuresis improved and creatinine progressively decreased (normal range within a month of surgery), being discharged one week after surgery.Postoperative ultrasound showed a significant decrease in bilateral ureterohydronephrosis, A year later, the patient remains asymptomatic without UTI.

Conclusion: The intravesicalization of the obstructed ectopic ureters by minimally invasive approach allows the resolution of acute urinary obstruction in a case of bilateral complex uropathy. The endoscopic approach is shown as an effective possibility in the diagnostic and therapeutic management of these patients.

Keywords: Ectopic ureter;Bilateral obstruction; Endoscopic drainage;Congenital malformation;Female fetus

\section{Introduction}

The presence of posterior urethral valves as a cause of oligoamnios is well known. Differential diagnosis should be established with other pathologies such as prune-belly syndrome, high-grade vesicoureteral reflux, bilateral obstructive megaureter, urethral atresia, obstruction of the anterior urethra, ureteroceles and less frequently the presence of an obstructed bilateral ectopic system [1]. The ectopic ureter is more frequent in girls, associated with a duplicated renoureteral system and rarely associated with bilateral obstruction of the urinary tract. We present an exceptional case of anhydramnios in a female fetus secondary to obstruction of a bilateral ectopic ureter and a duplicated left system with severe worsening of renal function.

\section{Material and Methods}

We present the case of a preterm newborn $(33+6$ weeks $)$ with adequate weight and a diagnosis in the third trimester of bilateral ureterohydronephrosis with double left system. Labor was induced at week 33 due to anhydramnios. At birth, a bladder catheter was placed and a renal ultrasound was performed. It showed a pielocalicial dilation (right side grade III, left side grade IV) and bilateral megaureter. A left double system was suspected with upper pole ectopic ureter and the lower pole draining in a bladder diverticulum. However, during her admission to the neonatology unit, she presented a progressive creatinine worsening up to $3.3 \mathrm{mg} / \mathrm{dL}$ despite bladder catheterization and an episode of urinary tract infection. 
An MRI was also performed to better define the anatomy. During cystoscopy, the right ectopic meatus could be identified in the upper area of the vagina, the left upper kidney ureter leading to the urethra and the left lower kidney ureter imprint in the bladder. By electrocoagulation a bilateral internal (neo-orifice) shunt was performed as a drainage method of both dilated systems (Figure 1).
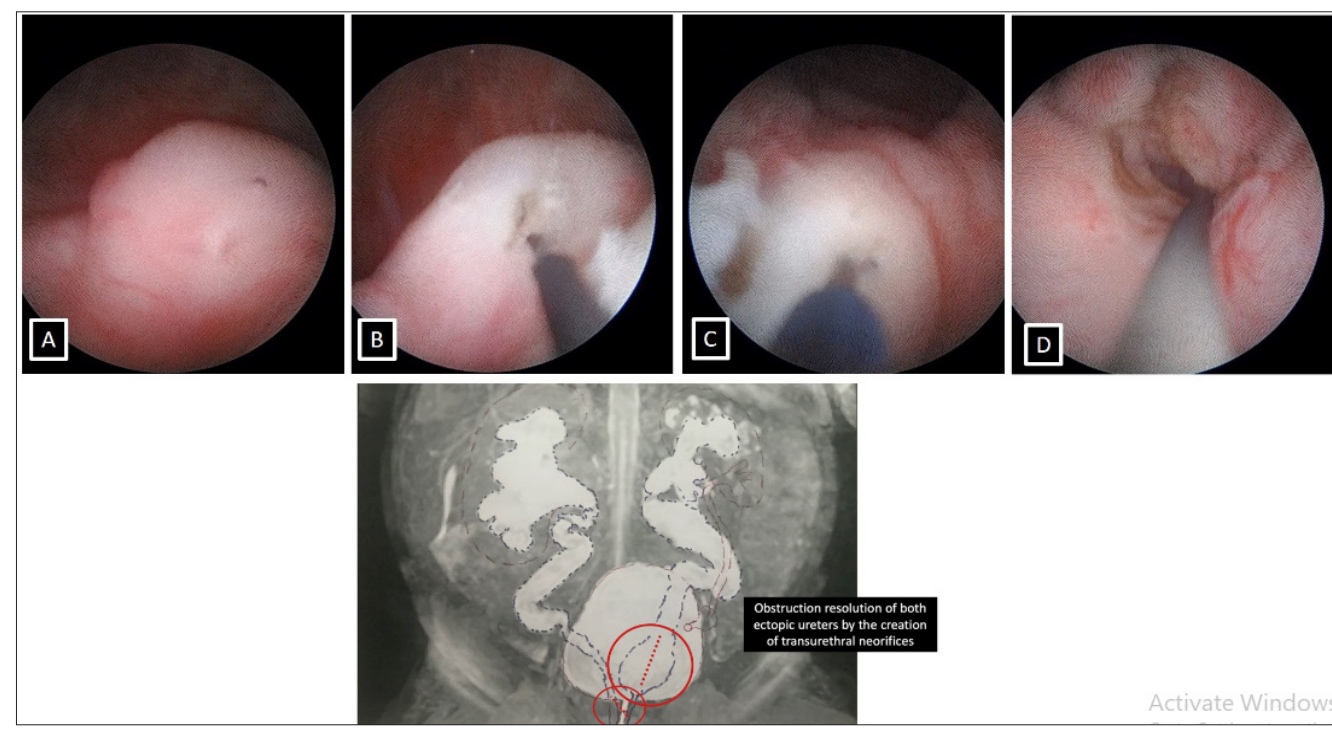

๑

Figure 1: Surgical images and correlation with the findings of the magnetic resonance.

A \& B: Bladder imprint of the right ectopic ureter and intravesicalization with electrocoagulation

C \& D: Left upper kidney ureter leading to the urethra and creation of trans-urethal neo-orifice.

\section{Result}

After surgery, the patient presented a clear clinical improvement with spontaneous diuresis and progressive decrease in renal function (normal range one month after surgery) (Figure
2). Likewise, a notable decrease in bilateral pielocalicial dilation was observed (Figure 3). After one year of follow-up, the patient is asymptomatic, has not presented urinary infections and maintains antibiotic prophylaxis due to vesicoureteral reflux to the lower kidney.

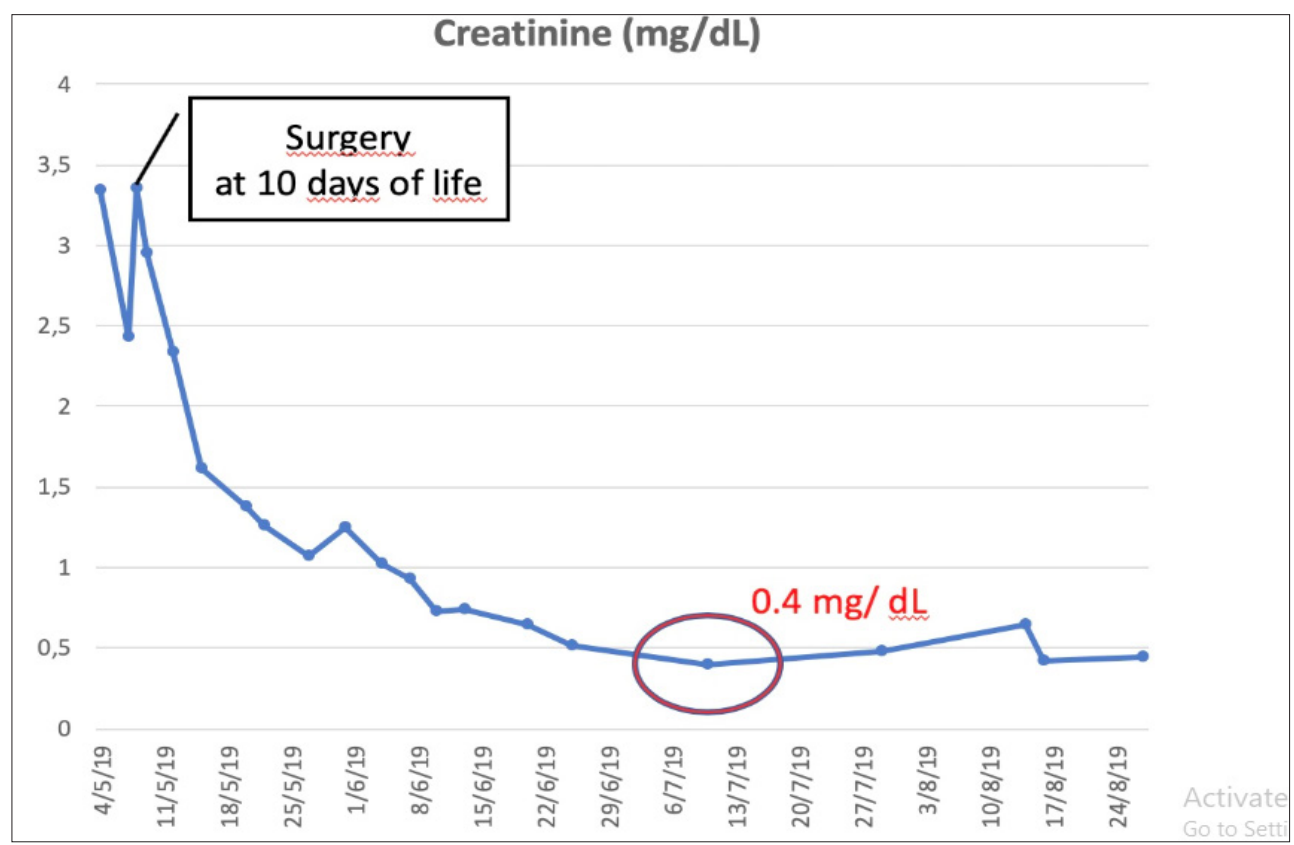

Figure 2: Evolution of creatinine values before and after surgery. 


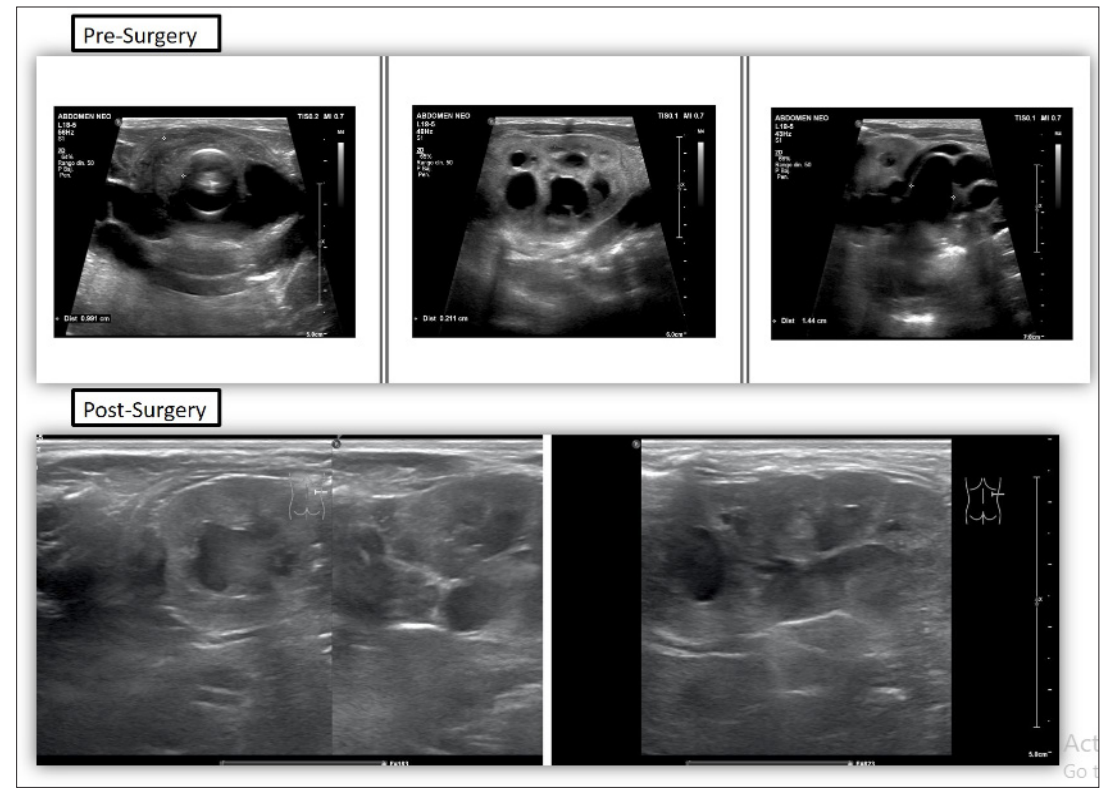

Figure 3: Improvement in ultrasound dilation after surgery.

\section{Discussion}

The ectopic ureter occurs in approximately 1 in 2000 newborns. It occurs more frequently in women and in $85 \%$ of cases it is associated with a pyeloureteral duplicity, usually depending on the ectopic ureter of the upper kidney [2]. In women, the most common sites of the ectopic meatus include the bladder neck, the proximal or distal urethra, the vestibule and the vagina. There have also been cases of ectopic ureteral orifices in the uterus and cervix [3]. Oligoamnios secondary to urethral pathology or bilateral ureteroceles has been described in children, which can lead to severe worsening of renal function and the need for renal transplantation in some cases [4]. However, there are no reports in the literature of bilateral ectopic ureteral obstruction in a female patient with progressive worsening of renal function and urgent need for bilateral drainage within a few days of life.

The treatment of obstructed ectopic ureter is controversial. Typically, an external shunt (nephrostomy or ureterostomy) is performed to relieve hydronephrosis and resolve ureteral obstruction acutely. However, in our practice in these cases we perform minimally invasive techniques to solve this problem, such as intravesicalization of the ureter or the creation of an endoscopic transurethral neorifice to resolve the obstruction of the ectopic ureter with results similar to the traditional treatment [5]. The form of endoscopic treatment used in this case allowed an improvement of the immediate obstruction and an improvement of the renal function, avoiding surgeries with greater morbidity in a newborn. It is a non-invasive procedure that allows adequate ureteral drainage in cases of severe obstruction with a high risk of uncontrollable urinary tract infection or uretero-pionephrosis.

The objective of this technique is to create a temporary internal drainage to control urinary infections, preserve the initial renal function and facilitate the baby to mature until a definitive surgery is proposed months later. It is reproducible, safe and does not invalidate other surgical options in case of failure or future definitive treatments, either by classical surgical approach, laparoscopy, laparo-assisted or with robotic surgery. So in conclusion, the endoscopic treatment by intravesicalization of the obstructed ectopic ureters allows an internal shunt to resolve the acute obstruction through a minimally invasive approach in a case of bilateral complex uropathy, reducing the morbidity and mortality associated with open surgeries or more invasive procedures.

\section{References}

1. Sherer DM, Hulbert WC (1995) Prenatal sonographic diagnosis and subsequent conservative surgical management of bilateral ureteroceles. Am J Perinatol 12(3): 174-177.

2. Martin MS, Garcia-Ripoll TJ, Ruiz SA, Rodriguez GV, Ferro RJ et al. (2008) Ectopic ureter as cause of pyonephrosis and urinary incontinence. Actas Urol Esp 32(2): 256-260.

3. Sirisreetreerux P, Lue KM, Michaud JE, Di Carlo HN, Gearhart JP (2016) Duplicated renal collecting system with ectopic ureter in female bladder exstrophy: A case report. Urology 89: 129-31.

4. Johnson MP, Danzer E, Koh J, Polzin W, Harman C, et al. (2018) Natural history of fetal lower urinary tract obstruction with normal amniotic fluid volume at initial diagnosis. Fetal Diagn Ther 44(1): 10-17.

5. Ortiz R, Parente A, Burgos L, Angulo JM (2017) Endoscopic urinary diversion as initial management of symptomatic obstructive ectopic ureter in infants. Front Pediatr 5: 208. 